\title{
Myrmécofaune arboricole associée aux couples Phragmanthera capitata (Sprengel) S. Balle/ hôte au verger de la chefferie de Ndogbong (Douala, Cameroun)
}

\author{
Ruth MONY ${ }^{1}$, Joseph Marie ONDOUA ${ }^{2}$, Siegfried Didier DIBONG ${ }^{2 *}$, \\ Joseph BOUSSIM ISSAKA $^{3}$ et AMOUGOU AKOA ${ }^{4}$ \\ ${ }^{I}$ Département de Biologie des Organismes Animaux, Faculté des Sciences, B. P. 24157, Université de \\ Douala, Cameroun. \\ ${ }^{2}$ Département de Biologie des Organismes Végétaux, Faculté des Sciences, B. P. 24157, Université de \\ Douala, Cameroun. \\ ${ }^{3}$ Laboratoire de Biologie et d'Ecologie Végétales, Université de Ouagadougou 03 B. P. 848, Ouagadougou 03, \\ Burkina Faso. \\ ${ }^{4}$ Département de Biologie et Physiologie Végétales, B. P. 812, Université de Yaoundé I, Cameroun. \\ *Auteur correspondant, E-mail: didierdibong@yahoo.fr
}

\section{RESUME}

Parmi les Loranthaceae, l'espèce Phragmanthera capitata s'est mieux adaptée aux conditions du milieu modifié par l'homme et aux arbres cultivés ou spontanés devenant un véritable fléau agronomique. La méthode de lutte ciblée plus prometteuse ne peut être satisfaisante que si les mécanismes qui régulent l'adaptation des Loranthaceae à leurs hôtes cultivés ou spontanés sont identifiés et maîtrisés. Dans cette optique, une étude de la myrmécofaune arboricole a été menée sur trois couples $P$. capitata/hôte au verger de la chefferie de Ndogbong. Auparavant, un inventaire exhaustif de tous les arbres parasités ou non et de la myrmécofaune du verger de la chefferie de Ndogbong (Douala) a été entrepris et toutes les touffes de $P$. capitata comptabilisés sur les arbres hôtes qui en possédaient. Tous les arbres du verger sont exotiques excepté Spondias mangifera. Le taux de parasitisme est de 42,85\%. Quatre espèces de fourmis (Crematogaster sp. 1, Crematogaster sp.2, Pheidole megacephala et Camponotus sp.) ont une activité fourragère notamment florifère sur les individus de $P$. capitata. Leur densité varie avec la floraison et semble liée à la chute des boutons floraux et des fleurs. Ces fourmis pourraient constituer des forces d'organisation et d'évolution exploitables dans la lutte biologique contre les Loranthaceae.

(c) 2009 International Formulae Group. All rights reserved.

Mots clés: Loranthaceae, myrmécofaune arboricole, plantes hôtes.

\section{INTRODUCTION}

Les Loranthaceae décrites par Balle (1982), sont des arbrisseaux épiphytes, chlorophylliens, qui vivent en hémiparasites sur les branches d'arbres et d'arbustes, sauvages ou cultivés. Ces plantes qui se présentent sous forme de touffes, sont ancrées dans le bois de l'hôte grâce à un suçoir qui permet d'établir des liaisons fonctionnelles avec l'appareil conducteur de l'hôte (Sallé et al., 1998). Le parasite prélève ainsi, l'eau et les sels minéraux et le complément de matière organique dont il a besoin. 
Les Loranthaceae appartiennent aux Angiospermes parasites qui ne représentent que $2 \%$ de l'ensemble des plantes à graines (Raynal-Roques et Paré, 1998). Mais dans certaines conditions, elles peuvent devenir de véritables fléaux. Leur répartition et les dégâts qu'elles occasionnent sont variables.

L'espèce Phragmanthera capitata (Sprengel) S. Balle, est largement répandue en Afrique, notamment au Cameroun, au Nigéria, au Gabon et en Côte d'Ivoire (Engone et al., 2005; Engone et Sallé, 2006; Dibong et al., 2008). Les cultures de rente telles que l'avocatier, le cacao, le café, les agrumes apparaissent fortement parasités et des pertes de rendement sont souvent considérables (Neumann et Sallé, 2003). La suppression mécanique des branches parasitées de l'hôte s'avère illusoire. La méthode de lutte ciblée plus prometteuse ne peut être satisfaisante que si les mécanismes qui régulent l'adaptation des Loranthaceae à leurs hôtes cultivés ou spontanés sont identifiés et maîtrisés.

Le présent travail consiste à étudier la biodiversité de la myrmécofaune arboricole associée aux couples $P$. capitata/hôte. Auparavant, un inventaire exhaustif de tous les arbres parasités ou non et de la myrmécofaune du verger de la chefferie de Ndogbong (Douala) a été entrepris et toutes les touffes de $P$. capitata comptabilisées sur les arbres hôtes qui en possédaient. L'activité fourragère des fourmis sur la floraison et la fructification de $P$. capitata été suivie pendant trois mois sur trois espèces hôtes (Citrus maxima, Psidium guajava et Theobroma cacao). Les activités des fourmis sont ensuite discutées.

\section{MATERIEL ET METHODES \\ Le site d'étude}

Le site d'étude est le verger du campement de la chefferie de Ndogbong, quartier situé au Nord-Est de la ville de Douala, Cameroun (Figure 1). Douala (latitude, $03^{\circ} 40-04^{\circ} 11^{\prime} \mathrm{N}$; longitude, 09 ${ }^{\circ} 16^{\prime}$ - 09.52' E; altitude, $13 \mathrm{~m}$ ) a un climat qui appartient au domaine équatorial d'un type particulier dit «camerounien» qui se caractérise par deux saisons avec une longue saison de pluies (au moins 9 mois), des précipitations abondantes (environ $4000 \mathrm{~mm}$ par an), des températures élevées $\left(26,7{ }^{\circ} \mathrm{C}\right)$ et stables. La moyenne minimale de température à Douala pour 30 années (1961-1990) est de $22,6{ }^{\circ} \mathrm{C}$ en juillet et la moyenne maximale de température de $32,3{ }^{\circ} \mathrm{C}$ en février. L'humidité relative de l'air reste élevée toute l'année et voisine de $100 \%$ (Dibong et al., 2009).

\section{Réalisation du relevé floristique}

Le verger prospecté a une superficie de 2 ha environ et est constitué essentiellement d'arbres fruitiers dont les fruits sont destinés à la consommation des propriétaires. Il est implanté dans une cacaoyère abandonnée et défrichée par la suite pour y planter des arbres utiles à l'alimentation.

Le travail a été réalisé sur le terrain de janvier à juillet 2009. L'objectif a consisté à identifier les Loranthaceae sur toutes les essences ligneuses de diamètre $(\mathrm{DBH}) \geq 5 \mathrm{~cm}$ parasitées du verger de la chefferie. On note pour chaque arbre parasité ou non: le diamètre à l'aide d'un mètre ruban et le nombre de touffes du parasite (si l'arbre en possède) en grimpant sur l'arbre à l'aide d'une échelle en bois.

\section{Récolte et identification de la myrmécofaune \\ Huit prélèvements matinaux des} fourmis ont été effectués sur trois couples $P$. capitata/hôte. Ces prélèvements ne sont pas faits systématiquement le long de la superficie du verger, mais sont motivés par la présence de $P$. capitata sur les arbres hôtes. Les ouvrières des fourmis qui fourragent sur les pantes sont récoltées, les zones d'ancrage ont été soulevées à l'aide de la machette pour capturer les fourmis dans les pores. Ces fourmis sont récoltées à l'aide de l'aspirateur à bouche (cas des ouvrières de taille moyenne) ou à l'aide d'une paire de pinces molles pour les ouvrières de plus grande taille et conservées dans des boîtes noires étiquetées contenant de l'alcool à $70{ }^{\circ} \mathrm{C}$ au laboratoire de 


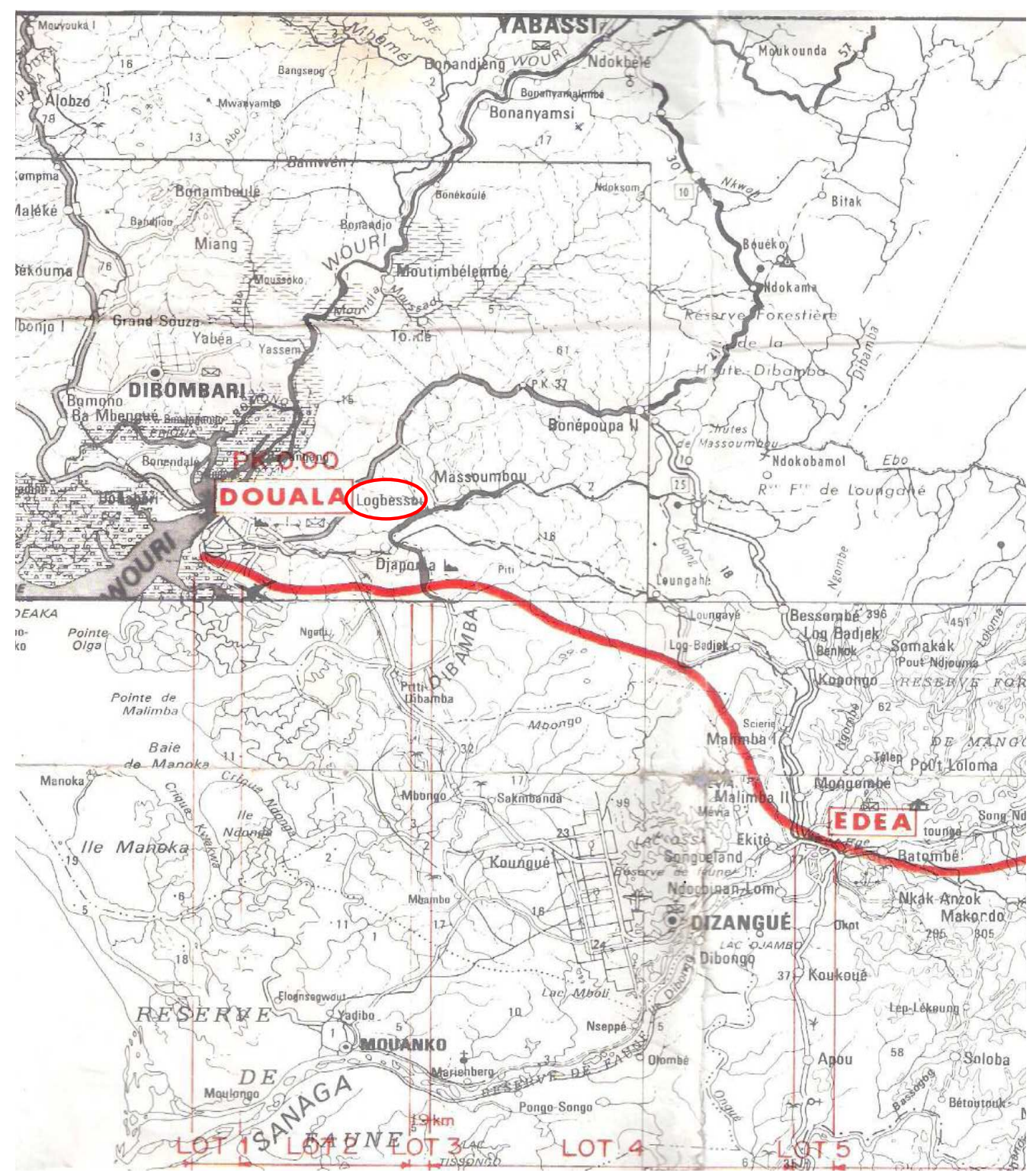

\section{Zone d'étude}

E : $1 / 500000$

Figure 1: Localisation de la région de Douala.

Biologie des Organismes Animaux de la Faculté des Sciences de l'Université de Douala.

L'identification des espèces de fourmis s'est faite en utilisant les clés d'identification de la base de données des fourmis africaines (www.antbase.org) et leur nomenclature approuvée par les spécialistes africains des fourmis Drs. Kenne Martin et Mony Ruth.

L'étude de l'activité fourragère des ouvrières sur les boutons floraux d'un nœud de rameau a été effectuée sur une Loranthaceae de chacune des trois espèces 
hôtes retenues: Citrus maxima, Psidium guajava, Theobroma cacao. Sur ces individus hôtes, des rameaux de Loranthaceae suffisamment proches du sol ont pu être marqués pour cette opération. Les mois de récolte des ouvrières sur les boutons floraux ont été avril, mai et juin 2009 et correspondent à la petite saison des pluies dans la zone d'étude. Les récoltes pour estimer la densité des individus des espèces ont toutes été effectuées le matin à 9 heures. Une grande boîte blanche contenant de l'alcool à $70^{\circ} \mathrm{C}$ est utilisée pour recueillir les fourmis balayées sur le nœud du rameau floral à l'aide d'un pinceau adapté à cet usage et vendu sur le commerce.

\section{Analyses statistiques des résultats}

Le pourcentage de parasitisme $(\mathrm{Pp}) \mathrm{du}$ verger est donné par la relation suivante:

$\mathrm{Pp}=$ (nombre d'arbres parasités / nombre d'arbres total) x 100

Pour déterminer la diversité des fourmis, l'indice de diversité $\alpha$ qui correspond à la diversité au niveau d'un habitat (arbre hôte ou Loranthaceae) a été utilisé. Il permet de comparer deux habitats. Les données sont exprimées en termes du nombre d'individus capturés pour chaque espèce ou genre. Cet indice a permis de déterminer:

- la richesse spécifique «S» qui est le nombre total des espèces dans un habitat;

- la fréquence relative $\mathrm{F}$ qui est le rapport du nombre d'individus $\mathrm{Ni}$ de l'espèce $\mathrm{i}$ sur le nombre total $\mathrm{N}$ d'individus récoltés multiplié par 100 pour avoir le résultat en pourcentage.

L'indice de dominance «d» de Berger et de Parker a été utilisé pour comparer la diversité spécifique de deux habitats. Le plus souvent, cet indice exprime l'importance de l'espèce la plus dominante. La faible valeur de «d» traduit une forte diversité. Cet indice est déterminé par la formule:

$\mathrm{d}=\mathrm{Nmax} / \mathrm{N}$

Nmax est le nombre d'individus de l'espèce la plus fréquente du milieu;

$\mathrm{N}$ est le nombre total des individus récoltés dans un habitat.
Les tests ANOVA LSD à partir du logiciel StatSoft, Inc. (2001) STATISTICA version 6.0 ont permis de comparer les densités des ouvrières de fourmis récoltées chaque mois.

\section{RESULTATS}

Inventaire floristique

$\mathrm{Au}$ total 49 individus hôtes, parasités ou non par les Loranthaceae ont été inventoriés sur le site et 19 portaient au moins un individu de Phragmanthera capitata. Les individus hôtes regroupent 11 espèces appartenant à 9 genres de 8 familles (Tableau 1). Parmi ces espèces, seule Spondias mangifera Willd. (Anacardiaceae) est subspontanée et a été maintenue sur pied après dégradation de la forêt. Les 48 autres individus hôtes recensés sur le site sont des plantes cultivées qui sont réparties de la façon suivante en fonction des familles: Sterculaceae, 16 individus de Theobroma cacao L. (cacaoyer); Anacardiaceae, 13 individus de Mangifera indica L. (manguier); Rutaceae, 10 individus de Citrus spp. (agrumes); Lauraceae, 3 individus de Persea americana Mill (avocatier); Myrtaceae, 3 individus de Psidium guajava Linn. (goyavier); Burseraceae, 1 individu de Dacryodes edulis (G. Don) H. Lam (safoutier); Clusiaceae, 1 individu de Garcinia cola Heckel (bitakola); Euphorbiaceae, 1 individu de Manniophyton fulvum Mull.-Arg.. L'espèce la plus abondante est Theobroma cacao avec 16 individus et celles moins abondantes avec un seul individu sont: Dacryodes edulis, Garcinia cola, Manniophyton fulvum.

Parmi les onze espèces recensées, quatre sont non parasitées (Citrus reticulata, Dacryodes edulis, Manniophyton fulvum et Mangifera indica) et sept parasitées ( $C$. maxima, C. sinensis, Garcinia cola, Persea americana, Psidium guajava, Spondias mangifera et Theobroma cacao). Les espèces ayant tous les individus parasités sont Garcinia cola, Persea americana, Psidium guajava, Spondias mangifera et Theobroma cacao. L'espèce ayant l'individu avec le plus 
Tableau 1: Parasitisme à Phragmanthera capitata (Sprengel) S. Balle des espèces ligneuses du verger de la chefferie de Ndogbong.

\begin{tabular}{|c|c|c|c|}
\hline Espèces hôtes & Individus sains & Individus parasités & TOTAL \\
\hline Anacardiaceae & & & 14 \\
\hline Mangifera indica $\mathrm{L}$. & 13 & 0 & \\
\hline Spondias mangifera Willd & 0 & 1 & \\
\hline Burseraceae & & & 1 \\
\hline $\begin{array}{l}\text { Dacryodes edulis (G. Don) H.J. } \\
\text { Lam }\end{array}$ & 1 & 0 & \\
\hline Clusiaceae & & & 1 \\
\hline Garcinia cola Heckel & 0 & 1 & \\
\hline Euphorbiaceae & & & 1 \\
\hline $\begin{array}{l}\text { Manniophyton fulvum Müll.- } \\
\text { Arg. }\end{array}$ & 1 & 0 & \\
\hline Lauraceae & & & 3 \\
\hline Persea americana Mill & 0 & 3 & \\
\hline Myrtaceae & & & 3 \\
\hline Psidium guajava Linn. & 0 & 3 & \\
\hline Rutaceae & & & 10 \\
\hline Cirus maxima Obsbeck & 0 & 1 & \\
\hline Citrus sinensis Arancio & 2 & 6 & \\
\hline Citrus reticulata Blanco & 0 & 1 & \\
\hline Sterculiaceae & & & 16 \\
\hline Theobroma cacao L. & 11 & 5 & \\
\hline TOTAL & 28 & 21 & 49 \\
\hline
\end{tabular}

grand nombre de touffes du parasite (hormis Spondias mangifera) est Psidium guajava avec 65 touffes et une circonférence de 89 $\mathrm{cm}$.

\section{Parasitisme de Phragmanthera capitata}

Le pourcentage moyen de parasitisme de Phragmanthera capitata dans le verger est de $42,85 \%$. Plusieurs remarques peuvent être formulées concernant le parasitisme de Phragmanthera capitata dans le site:

- le milieu prospecté est le résultat des activités anthropiques et héberge un arbre domestiqué de l'espèce Spondias mangifera présente dans la forêt originelle;

- le rapport de parasitisme du nombre des individus non parasités et du nombre d'individus parasités est égal à 1,33 et montre que l'inversion de celui-ci sera difficilement atteinte. En effet, le manguier résistant au parasitisme (obs. pers.) a treize individus non parasités dans le site. L'évolution du parasitisme sera seulement perceptible par le cumul des touffes de $P$. capitata sur les arbres déjà parasités;

- les habitudes alimentaires et culturelles se traduisent dans cette flore. La chefferie appartient à la tribu «Bassa» et les fruits de Spondias mangifera sont très appréciées des propriétaires;

- les disséminateurs aviaires sont présents et infectent aussi bien les arbres de petits que de gros diamètres bien qu'ils préfèrent les individus d'avocatier et de goyavier: le type de fruit influence aussi le comportement aviaire. L'oiseau s'installe avec la baie sur un rameau voisin de la touffe de Loranthaceae, tandis qu'elle maintient la baie contre l'écorce avec sa patte, elle retire et avale la partie externe de la pulpe adhérant aux téguments. La graine ainsi dégagée reste collée sur l'écorce, parfaitement intacte et prête à germer. 
Tableau 2: Fréquences relatives des fourmis pour 214 individus récoltées après huit prélèvements matinaux.

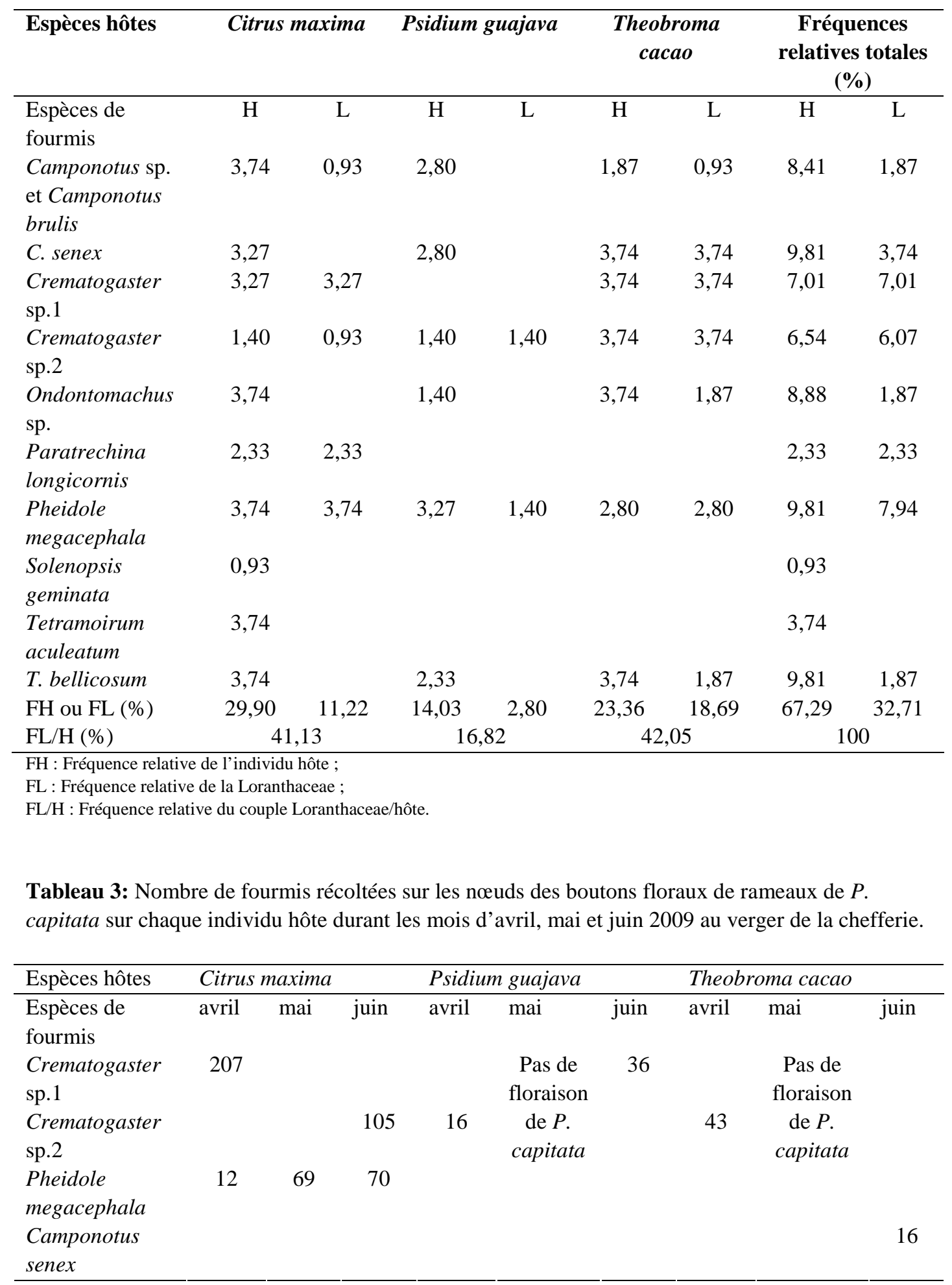




\section{Inventaire de la myrmécofaune}

Au total 10 espèces de fourmis ont été recensées sur les trois couples $P$. capitata/hôte retenus. Les arbres hôtes appartiennent à trois espèces: Citrus maxima, Psidium guajava et Theobroma cacao. Les espèces de fourmis sont réparties en trois sous-familles (Tableau 2):

- Ponerinae avec une seule espèce, Odontomachus sp.;

- Formicinae avec deux espèces, Camponotus sp. et Camponotus senex;

- Myrmicinae est la plus diversifiée et comptent sept espèces, Cremastogaster sp.1, Crematogaster sp.2, Paratrechina longicornis, Pheidole megacephala, Solenopsis geminata, Tetramorium bellicosum, Tetramorium aculeatum. Parmi celles-ci, certaines sont terricoles et d'autres sont arboricoles. Cette stratification des sites de nidification influence l'activité fourragère (Tableau 3).

Sur huit prélèvements matinaux, 214 individus ont été comptés dont 70 sur $P$. capitata et 144 sur les arbres hôtes soient des fréquences relatives respectives de $32,71 \%$ et $67,29 \%$. Trois espèces sont dominantes sur les arbres hôtes: Camponotus senex, Pheidole megacephala et Tetramorium bellicosum. Leur indice de dominance $\mathrm{dH}$ (pour les espèces hôtes) de Berger et Parker est de 0,098 . L'espèce de fourmi dominante sur $P$. capitata est Pheidole megacephala avec un indice de dominance dL (pour les Loranthaceae) de Berger et Parker de 0,079. Les valeurs de cet indice sont proches de zéro et traduisent une forte diversité ou une dominance négligeable de ces fourmis soit sur les arbres hôtes soit sur $P$. capitata. L'espèce de fourmi dominante à la fois sur Phragmanthera capitata et son hôte, Citrus maxima est Pheidole megacephala. La fréquence relative calculée est de $3,74 \%$ dans les deux cas. Paratrechina longicornis est la seule espèce spécifique de Citrus maxima dans le verger.

Deux espèces de fourmis présentes sur les arbres hôtes sont absentes sur la Loranthaceae qui les parasite: Solenopsis geminata et Tetramorium aculeatum sur Citrus maxima. Leur fréquence relative est respectivement de 0,93 et $3,74 \%$. Cet agrume est aussi le seul arbre hôte fréquenté par toutes les fourmis avec une fréquence relative de $29,90 \%$. Les autres arbres hôtes ont des fréquences relatives de 23,36 et $14,06 \%$ respectivement pour Theobroma cacao et Psidium guajava. La fréquence relative la plus élevée pour $P$. capitata est de $18,69 \%$ sur Theobroma cacao qui pourtant montre une fréquence significativement $(\mathrm{p}<0,01)$ moins élevée que $C$. maxima. Ainsi, la proportion des fourmis sur Phragmanthera capitata ne varie pas toujours dans le même sens que celle des individus hôtes. Deux espèces de fourmis sont présentes sur les trois couples Loranthaceae/hôte. D'une part Crematogaster sp.2 avec une fréquence relative de 3,74\% sur le couple $P$. capitata/Theobroma cacao et Pheidole megacephala et d'autre part avec la même fréquence relative sur le couple $P$. capitata/C. maxima, les ouvrières fourrageant sur tous les individus hôtes. Cette activité fourragère est marquée par la prédominance des ouvrières co-dominantes en fonction des mois de récolte choisis (Tableau 3).

Les observations répétées des nœuds ont montré que les ouvrières sont isolées ou absentes sur les nœuds lorsque les fleurs sont épanouies et que les fruits sont apparus. Cependant sur les rameaux floraux, des colonies d'ouvrières ont été signalées sur les nœuds des boutons floraux et les comptages effectués (Tableau 3). Les tests de variance LSD de comparaison entre les mois de récolte des ouvrières ont permis de déceler des différences de densité hautement significatives $(p<0,001)$.

\section{DISCUSSION}

La plupart des individus de Spondias mangifera en âge de produire rencontrés hors site dans la région de Douala sont également parasités par Phragmanthera capitata. Il en est de même pour les espèces Persea americana et Psidium guajava. Les travaux de Carlo et al. (2003) ont montré que les frugivores préfèrent se nourrir des fruits d'une 
ou de plusieurs espèces hôtes spécifiques et les visitent plus que d'autres. Cette hypothèse est renforcée par le parasitisme de l'avocatier par $P$. capitata dans la région de Douala (Dibong et al., 2008) et Yaoundé (Sonké et al., 2000).

Les fourmis sont en général considérées comme étant des prédateurs (consommateurs secondaires). Toutefois, les liquides sucrés tels que nectaires extrafloraux et le miellat d'Homoptères représentent une part importante du régime alimentaire chez les espèces arboricoles et chez quelques espèces terricoles (Lévieux, 1976a; Dejean et Corbara, 1990; Dejean et Suzzoni, 1997a; Völkl et al., 1999; Orivel et Dejean, 1999). Cette prédation permet le ravitaillement de la colonie en matières protéiques (Hölldobler et Wilson, 1990).

Suivant les espèces de fourmis, les ouvrières peuvent occasionner des lésions sur les parties souterraines et aériennes des plantes cultivées (bourgeons, feuilles, fleurs et fruits) appartenant à plusieurs familles pour exploiter directement de la sève ou pour ronger les tissus internes du végétal. L'ampleur des dégâts occasionnés peut varier des simples lésions au découpage en morceaux des parties molles du végétal, provoquant ainsi la mort de la plante (Kenne et al., 1999).

Les espèces de fourmis moissonneuses appartiennent aux sous-familles de Ponerinae, Myrmicinae et Formicinae (Hölldobler et Wilson, 1990). Ce sont des fourmis qui récoltent des graines et les entassent dans leurs nids sous forme de réserves de nourriture (Hölldobler, 1976). Dans le milieu, les graines récoltées en grand nombre sont celles des espèces végétales les plus abondantes qui peuvent perdre jusqu'à $90 \%$ de leurs graines (Beattie, 1989; Inouye et al., 1980). Tel n'est pourtant pas le cas pour les Loranthaceae notamment $P$. capitata dont les fruits sont des pseudobaies. Celles-ci possèdent classiquement un péricarpe très développé où le mésocarpe est la composante essentielle et constitue la viscine. Ainsi, bien que très riche en polysaccharides (Gedalovich et al., 1988), sa consistance très visqueuse (Edouard et Raynal-Roques, 1989) constitue un piège mortel pour les ouvrières des fourmis. Néanmoins, la majorité des espèces de fourmis moissonneuses connues sont des Myrmicinae du genre Pheidole (Kenne et al., 1999). Ces dernières sont caractérisées par des colonies populeuses (Erickson et Mark, 1972) et une forte agressivité vis-à-vis des compétiteurs inter et intraspécifiques (Hölldobler et Wilson, 1990). Les fourmis du genre Pheidole s'attaquent beaucoup aux espèces végétales abondantes (Kenne et al., 1999). Tel est le cas de $P$. capitata sur Citrus maxima au verger dont la chute de nombreux boutons floraux et des fleurs serait due aux ouvrières du genre Pheidole. Cet aspect pourrait être exploité avantageusement dans le cadre de la lutte biologique contre $P$. capitata.

Dans la sous-famille des Myrmicinae, Solenopsis geminata et Tetramorium spp. se nourrissent de pétales, du tissu ovarien des fleurs et fruits des plantes pérennes telles les citronniers et cacaoyers (Veeresh, 1984; obs. pers.). Les observations faites sur $P$. capitata au verger sont en accord avec les dommages occasionnés aux plantes par des fourmis arboricoles foreuses $\mathrm{du}$ bois du genre Camponotus (Lévieux, 1976b) et des fourmis cartonnières du genre Crematogaster (Prins et al., 1990). Chez les fourmis foreuses du genre Camponotus, les nids sont formés de galeries creusées dans le bois vivant (Kenne et al., 1999). Ces galeries forment un réseau impressionnant dans les suçoirs des Loranthaceae, Angiospermes hémiparasites. L'activité de ces fourmis entraînent une baisse de rendement de la plante ou le desséchement de la partie occupée (Kenne et al., 1999). Chez Crematogaster, certaines sont foreuses et occupent les parties desséchées de la plante hôte et/ou de la Loranthaceae. D'autres construisent des nids en carton à partir des particules des feuilles prélevées sur les couples Loranthaceae/hôte.

De nombreuses fourmis exploitent des Homoptères et /ou les larves de Lépidoptères Lycaenidae (Banks et al., 1991). Ces associations entraînent des dégâts 
considérables sur les organes végétatifs des plantes (Dejean et al., 1997b; Dejean et al., 1996). Les espèces de fourmis associées aux insectes piqueurs des plantes se rencontrent chez les Formicinae, Myrmicinae et Ponerinae (Kenne et Dejean, 1999; Maschwitz et al., 1987). Ces associations entraînent fréquemment la prolifération des Homoptères et en conséquence les dommages sur les plantes hôtes (Samways, 1990). Les fourmis $\mathrm{du}$ genre Crematogaster protègent des Pseudococcidae sur les cacaoyers, jouant un rôle néfaste dans les plantations cacaoyères (Dejean et al., 1991). Ainsi, ces dommages sur les plantes épiphytes notamment les Loranthaceae mériteraient d'être aussi étudiés pour promouvoir la lutte biologique.

\section{Conclusion}

Le régime alimentaire de nombreuses espèces de fourmis leur permet d'atteindre des densités de population élevée marquant une co-évolution entre les fourmis et les plantes (Davidson, 1988).

De part l'étroitesse des relations que les fourmis entretiennent avec les plantes, elles ont colonisé les milieux arboricoles. L'apparition de la vie arboricole est donc un caractère développé secondairement (Orivel et Dejean, 1999).

La notion de mosaïque est apparue à la suite des études sur les plantations de cacaoyer au Ghana (Majer, 1976). Elle se définit comme l'ensemble des espaces occupés par les territoires des colonies de fourmis dominantes. Les fourmis dominantes Camponotus senex, Crematogaster sp.1, Crematogaster sp.2 et Pheidole megacephala influencent fortement la distribution et le nombre des autres insectes du verger de la chefferie. La mosaïque de fourmis dans ce milieu où les arbres cultivés sont parasités par Phragmanthera capitata représente d'importantes forces d'organisation et d'évolution qu'il convient d'approfondir dans la recherche d'une méthode de lutte ciblée adéquate au parasitisme des Loranthaceae.

\section{REMERCIEMENTS}

Notre profonde gratitude à MBODY Samson, notable à la chefferie de Ndogbong.

\section{BIBLIOGRAPHIE}

Balle S. 1982. Loranthacées, Flore du Cameroun, vol. 23, Satabié B., Leroy J. F., Yaoundé, Cameroun. 82 p.

Banks WA, Adams CT, Lofgren CS. 1991. Damage to young Citrus trees by the red imported fire ant (Hymenopteral Formicidae). J. Entomol., 84: 241-246.

Beattie AJ. 1989. The effect of ants on grasslands. In Grassland Structure and Function: California Annual Grassland, Huenneke LF, Mooney H (eds). Kluwer Academic Publishers: Dordrecht, Netherlands; 105-116.

Davidson DW. 1988. Ecological studies of Neotropical ant gardens. Ecology, 69: 1138-1152.

Dejean A, Corbara B. 1990. L'alimentation sucrée des larves chez Pachycondyla villosa (Formicidae, Ponerinae). Biol. Behav., 15: 117-124.

Dejean A, Nkongmeneck B, Corbara B, Djiéto-Lordon C. 1991. Impact des fourmis arboricole sur une prolfération d'Achaea catocaloides (Lepidoptera, Notuidae) dans une cacaoyère du Cameroun. Acta Oecol., 12: 471-488.

Dejean A, Ngnegueu PR, Bourgoin T. 1996. Trophobiosis between ants and Peregrinus maidis (Hemiptera, Fulgoromorpha, Delphacidae). Sociobiology, 28: 183-195.

Dejean A, Suzzoni JP. 1997a. Surface tention strengths in the service of a ponerine ant: a new kind of nectar transport. Naturwissenschaften, 84: 76-79.

Dejean A, Djiéto-Lordon C, Durand JL. 1997b. Territoriality of ant species induced by mutualistic tettigometrid: a new case of fig protection. Ecoscience, 4 : 446-453.

Dibong SD, Din N, Priso RJ, Taffouo VD, Fankem H, Amougou A. 2008. Parasitism of host trees by the Loranthaceae in the region of Douala 
(Cameroon). African Journal of Environmental Science and Technology, 2(11): 371-378.

Dibong SD, Engone Obiang NL, Din N, Priso RJ, Taffouo VD, Fankem H, Sallé G, Missoup AD, Amougou A. 2009. An assessment on the uses of Loranthaceae in ethno pharmacology in Cameroon: A case study made in Logbessou, North of Douala. Journal of Medicinal Plants Research, 3(8): 592-595.

Edouard JA, Raynal-Roques A. 1989. Le fruit des guis (Loranthaceae et Viscaceae): utilité pour la taxonomie et le mode de dissémination par les oiseaux. Bull. Mus. Natn. Hist. Nat., Paris, 11: 309-314.

Engone Obiang NL, Paré J, Duredon J, Sallé G. 2005. Germination et développement de la plantule d'Helixanthera mannii (Oliv.) Danser (Loranthaceae) sur cacaoyer (Theobroma cacao L.) au Gabon. Revue de Cytologie et de Biologies Végétales- Le Botaniste, 29: 1321.

Engone Obiang NL, Sallé G. 2006. Faut-il éradiquer Phragmanthera capitata, parasite des hévéas en Afrique? $C . R$. Biologies, 329: 185- 195.

Erickson JM, Mark 1972. Recapture techniques for population estimates of Pogonomyrmex ant colonies: an evaluation of the 32P technique. Annals Ent. Soc. Am., 62: 1274-1284.

Gedalovich E, Kuijt JL, Carpitas NC. 1988. Chemical composition of viscin, an adhesive involved in dispersal of the parasite Phoradendron californicum. Physiol. Mol. Plant Path., 32: 61-76.

Hölldobler B. 1976. Recruitment behavior, home range orientation and territoriality in harvester ants, Pogonomyrmex. Behav. Ecol. Sociobiol., 1: 1-44.

Hölldobler B, Wilson EO. 1990. The Ants. The Belknap Press of Haward University Press: Cambridge, Massachusetts, USA; $732 \mathrm{p}$.

Inouye RS, Byers GS, Brown JH. 1989. Effect of predation and competition on survivorship, fecundity and community structure of desert annuals. Ecology, 61: 1344-1351.

Kenne M, Dejean A. 1999. Diet and foraging activity in Myrmicaria opaciventris (Hymenoptera, Formicidae: Myrmicinae). Sociobiology, 33: 171-184.

Kenne M, Corbara B, Dejean A. 1999. Impact des fourmis sur les plantes cultivées en milieu tropical. Année Biol., 38: 195-212.

Lévieux J. 1976a. La nutrition des fourmis tropicales, IV: Cycle d'activité et régime alimentaire de Platythyrea conradti Emery (Hymenoptera, Formicidae, Ponerinae). Ann. Univ. Abidjan, ser. E, 9: 352-365.

Lévieux J. 1976b. Etude de la structure du nid de quelques espèces terricoles de fourmis tropicales. Ann. Univ. Abidjan, ser. C 12: 23-33.

Maschwitz U, Fiala B, Dolling WR. 1987. New trophobiotic symbioses of ants with South East Asian bugs. J. Nat. Hist., 21: 1097-1107.

Neumann U, Sallé G. 2003. Mécanismes de défense des plantes contre les Angiospermes parasites. C.R. Agric. Fr., 86(8): 85-96.

Orivel J, Dejean A. 1999. L'adaptation à la vie arboricole chez les fourmis. L'Année Biologique, 38: 132-148.

Prins AJ, Robertson HG, Preins A. 1990. Pest ants in urban and agricultural areas of southern Africa. In Applied Myrmecology; A world Perspectiva, Vander Meer RK, Jaffe K, Cedeno A (eds). Westview Press: Boulder, San Francisco, Oxford; 25-33.

Raynal-Roques A, Paré J. 1998. Biodiversité des Phanérogames parasites: leur place dans la classification systématique. Adansonia, 20: 313-322.

Sallé G. 1983. Germination and establishment of Viscum album L. In The Biology of Mistletoes, Calder M, Bernhardt P. (eds). Academic Press: Australia; 145-159. 
Sallé G, Tuquet C, Raynal-Roques A. 1998. Biologie des Phanérogames parasites. $C$. R. Soc. Biol., 192: 9-36.

Samways MJ. 1990. Ant assemblage structure and ecological management in Citrus and subtropical fruit orchads in Southern Africa. In Applied Myrmecology. A world Perspective, Vander Meer RK, Jaffe K, Cedeno A (eds). Westview Press: Boulder, San Francisco, Oxford; 570-587.

Sonké B, Kenfack D, Tindo M. 2000. Parasitisme de l'avocatier (Persea Americana Mill, Lauraceae) par les
Loranthaceae de la région de Yaoundé (Cameroun). Fruits, 55: 325-331.

Veeresh GK, Gubbaiah 1984. A report on the «crazy ant»(Anolepsis longipes (Jerdon)) menace in Karnatala. J. Soil Biol. Ecol., 4: 65-73.

Völkl W, Woodring J, Fisher M, Lorenz MW, Hoffmann KH. 1999. Ant-aphid mutualisms: the impact of honeydew production and honeydew sugar composition on ant preferences. Oecologia, 118: 483-491. 